\title{
663 MEDIA BASED ON THE METABOLIC COMPOSITION OF TUMOR INTERSTITIAL FLUID REVEALS PERSISTENT T CELL DYSFUNCTION INDUCED THROUGH ARGININE DEPRIVATION AND EXPOSURE TO THE ONCOMETABOLITE PHOSPHOETHANOLAMINE
}

${ }^{1}$ Yupeng Wang* ${ }^{2}$ Chufan Cai, ${ }^{1}$ Dayana Rivadeneira, ${ }^{2}$ Alexander Muir, ${ }^{1}$ Greg Delgoffe. ${ }^{1}$ University of Pittsburgh, Pittsburgh, PA, United States; ${ }^{2}$ University of Chicago, Chicago, United States

Background While CD8 T cells are crucial for anti-tumor immunity, tumor infiltrating CD8 T cells encounter stressors which deviate their differentiation to a dysfunctional, exhausted phenotype. $\mathrm{T}$ cell functions are closely regulated by $\mathrm{T}$ cell metabolism, and the dysfunctional vasculature in tumor tissues and the deregulated metabolism of tumor cells lead to depletion of nutrients and accumulation of metabolic wastes in the tumor microenvironment (TME). Thus, the unbalanced levels of the nutrients and the metabolic wastes might skew the metabolism of $\mathrm{T}$ cells thus contributing to $\mathrm{T}$ cell dysfunction.

Methods Ovalbumin-specific OT-I cells were activated with SIINFEKL/IL2 and cultured with IL2. The tumor interstitial fluid media (TIFM) was formulated based on the concentrations of the metabolites measured in the tumor interstitial fluid of pancreatic ductal adenocarcinoma. ${ }^{1}$ Purified arginine and phosphoethanolamine (PEtn) were used to change their levels in TIFM/RPMI1640 culture. Expression level of cytokines and PD-1 was measured by flow cytometry.

Results We sought to determine how $\mathrm{T}$ cells would differentiate, in vitro, if they were exposed only to the metabolites present in the TME. Using media formulated to model the metabolic composition of tumor interstitial fluid (TIFM), ${ }^{1}$ we show that CD8 $\mathrm{T}$ cells develop features of exhausted $\mathrm{T}$ cells in the TIFM culture: reduced proliferation, increased expression of PD-1 and decreased cytokine production. Using 'dropout' and 'add-back' approaches, we found arginine levels as a major contributor to the proliferation defect observed in TIFM-cultured T cells. Arginine was sufficient to restore proliferative capacity to $\mathrm{T}$ cells cultured in TIFM, but had no effect on the inhibited cytokine production. We then asked which metabolites were enriched in the TIFM, finding that PEtn, an intermediate in the ethanolamine branch of the Kennedy pathway and an oncometabolite enriched in the interstitial of many solid tumors, up-regulates PD-1 expression and compromises the cytokine production of the cells in culture. Depletion of Pcyt2, the metabolizing enzyme of PEtn and the rate limiting enzyme in the Kennedy pathway, makes CD8 T cells resistant to the effects of PEtn.

Conclusions Our data shows that the metabolic environment in the TME can be recapitulated in vitro and is sufficient to drive $\mathrm{T}$ cell dysfunction. Arginine depletion acts as a major inhibitor of $\mathrm{T}$ cell proliferation in the TME, but the oncometabolite PEtn drives a hypofunctional effector fate of $\mathrm{T}$ cells. Targeting PEtn metabolism via Pcyt2 depletion or inhibition is a potential target to reinvigorate $\mathrm{T}$ cells and enhance antitumor immunity.

\section{REFERENCE}

1. Sullivan MR, Danai LV, Lewis CA, Chan SH, Gui DY, Kunchok T, Dennstedt EA, Vander Heiden MG, Muir A. Quantification of microenvironmental metabolites in murine cancers reveals determinants of tumor nutrient availability. Elife 2019;;8: e44235. doi: 10.7554/eLife.44235. PMID: 30990168; PMCID: PMC6510537. 